\title{
IVth International Symposium for Health Professionals in Rheumatology
}

\section{Harrogate, 10th-12th June 1992.}

\begin{abstract}
The symposium was organised at the request of the Standing Committee for Health Professionals of the European League Against Rheumatism. The Organising Committee had the support of the University of Leeds and the British Health Professionals in Rheumatology.
\end{abstract}

\section{Plenary session I : Surgery of the upper limb in arthritis}

\section{I.1. Arthritis surgery of the shoulder and elbow.}

R.J. Newman. Academic Unit of Orthopaedic Surgery, St James's University Hospital, Leeds, LS9 7TF, UK.

Until approximately 25 years ago surgery was regarded as having little to offer in the management of rheumatoid arthritis of the shoulder and elbow. However, the advent of more refined clinical and radiological methods for diagnosis together with development of suitable prostheses has changed the whole scene and shoulder and elbow surgery should now be regarded as a routine part of the rheumatological surgeon's armamentarium for dealing with patients with the ravages of arthritis.

With regard to the shoulder the cornerstone of management should be the precise pre-operative assessment of the degree of pathological involvement of the individual subunits of the shoulder girle and the extent of surgery should be "tailored" to the individual patient. The results of reconstructive surgery using various types of shoulder arthroplasty will be discussed as will be the role of accurately placed steroid injection therapy based upon sensory testing with local anaesthetic.

Surgery can also be offered for the patient with either early or late involvement of the elbow joint with arthritis and the role of arthroplasty will be discussed.

Results of even the most sophisticated and expertly performed upper limb joint surgery in the arthritic patient can always be improved by an intensive and meticulous rehabilitation programme (both pre-operatively and post-operatively), the prescription of appropriate aids and appliances and the optimisation of medical therapy.

\section{I.2. Surgery of the wrist and hand in arthritis.}

M.Flowers. Leeds General Infirmary Gt George Street, Leeds, LS1 3EX, UK.

Appropriately planned surgery in the arthritic hand and wrist can make a profound difference to the patient, but careful preoperative assessment is essential.

1. Why do patients want surgery?

Evaluation involves good clinic atmosphere, where the patient's needs can be listened to. The Occupational Therapist has a key role. Precise and realistic objectives need to be set by surgeons and the patient together.

2. Can surgery be avoided, or deferred?

3. Why do deformities develop ?

A clear understanding of the mechanisms operating in each patient is important if reconstructive surgery is to be successful.

4. Which operation is "best buy"?

Experience dictates likely outcome, and risk taking is unwise. Some examples of recommended procedures, prophylactic, currative, reconstructive and salvage.

\section{Parallel session II.A : Occupational therapy}

II.A.1. Activities of daily living (ADL) and rheumatic disablement : the challenge to occupational therapy.

A. Tennant ${ }^{*}$, E. Badley ${ }^{* *}$. ${ }^{*}$ Rheumatology \& Rehabilitation Research Unit, University of Leeds ; ${ }^{*}$ University of Toronto.

Occupational therapy (OT) is aimed at improving, or at least maintaining independence of clients. What sort of demand for OT, arising from the rheumatic diseases, is likely to exist in the community? In 1986, 597 respondents aged 16 years and over with rheumatic disability were given a face-to-face interview as part of a large scale community survey. This paper examines the prevalence of difficulty across a wide range of ADL, and considers the likely implication for OT intervention. 79.1 per 1000 adults were found to be disabled in association with the rheumatic diseases $(95 \% \mathrm{CI}:+/-2.6)$. The most common reported difficulty $(70 \%)$ was with shopping, giving a prevalence of $55 / 1000$ adults. Cutting toenails was reported as difficult by over three-fifths, giving a prevalence of $40 / 1000$. Half of the respondents found picking items up off the floor difficult, giving a prevalence of 40/1000.

Some activities relate closely to overall level of dependence. For example, only 2 in 5 of those disabled but independent reported difficulty with picking things up off the floor, compared to almost all (95\%) of those needing continuous help or supervision. Other activities, for example carrying shopping, are found difficult by many, even those maintaining independence. About one-quarter of respondents found personal activities like washing all over, or dressing, difficult, but these activities were associated with dependency, and only one in ten of those independent reported such difficulty.

Factor analysis of ADLs suggest that light housework, including food preparation, is a major grouping of ADLs; that heavy housework is another group, and that dexterity and within-thehome mobility are others. The implications of these groups for the role of the OT, particularly in support of home care services, will be examined. 
VI.A.5. Long-term effects of group education for patients with rheumatoid arthritis.

E. Taal*, E.R. Seydel*, R.P. Riemsma*, H.L.M. Brus**, J.J. Rasker*,**, O. Wiegman*. *Department of Psychology, University of Twente, P.O.B. 217, 7500 AE Enschede ; ${ }^{*}$ Department of Rheumatology, Hospital Medisch Spectrum Twente, P.O.B. 50000, 7500 KA Enschede, the Netherlands.

Patients with rheumatoid arthritis (RA) must adjust their exercise, rest and medication to dialy disease activity. This presupposes adequate treatment and support by health professionals. How patients themselves cope with the consequences of their disease also has a major impact on their health status. Patient education can help patients to attain the necessary management behaviours. We developed a group training program based on Bandura's self-efficacy theory and a pilot study in 86 RA patients.

Our program is partially modelled after the 'Arthritis SelfManagement Program' developed in the USA by Lorig. The program consists of 5 weekly sessions of 6 to 8 patients with two trained expert groupleaders. Goal of the program is to increase self-efficacy and independence of patients in managing their health problems. Program content included information on RA and treatment, self-management and problem-solving, pain-management, relaxation and physical exercises, communication skills, coping with depression. Emphasis in the program lies on practising skills and not on knowledge transfer. Contracts are used to stimulate patients to practise at home. All patients receive a self-help guide.

The program has been evaluated in a field-experiment with an experimental group $(n=35)$ and a control group $(n=35)$. Outcome was assessed with mailed questionnaires, clinical assessment by physician and laboratory tests. Assessments are performed at baseline (before intervention) at 6 weeks (directly after intervention), at 4 months and 14 months after baseline. Directly after intervention we find positive effects of the group training on function, practice of relaxation and physical exercises, self-management behaviour, outcome expectations, selfefficacy and knowledge. At 14 months we still find long term effects on practice of physical exercise, self-efficacy and knowledge. Short term effects on function, practice of relaxation exercises, self-management behaviour and outcome expectations are not maintained at 14 months. Although the program is partly successful, it is concluded that to maintain changes it is necessary to hold booster sessions after the end of the 5-week program or to introduce other forms of feedback or support.

VI.A.6. Individual education to people with rheumatoid arthritis.

R.P. Riemsma*, E. Taal*, H. Brus**, O. Wiegman*, J.J. Rasker*,**. ${ }^{*}$ Department of Psychology, University of Twente, P.O.B. 217, 7500 AE Enschede ; ${ }^{*}$ Department of Rheumatology, Medisch Spectrum Twente, P.O.B. 50000, 7500 KA Enschede, the Netherlands.

In the treatment of persons with rheumatoid arthritis (RA) several health care workers are involved. Both patients and health care workers experience the lack of coordination and continuity in education and counselling as a problem. The aim of our study is to develop a model of coordinated education by different health professionals; thus enabling the patient to manage the problems during the course of their disease.

Based on our experience in different fields of health care we have developed a model for individual education of people with rheumatoid arthritis. A prerequisite is that the education and counselling activities should not place an additional burden on the shoulders of health professionals. The rheumiatologist, who has the first contact with the patient, will hand out a self-help-guide after the first consult. This guide includes concrete directions and exercises explaining how to deal with their psycho-social and physical problems. Other health professionals (like physiotherapists, community nurses and district nurses) are asked to adapt their activities to this self-helpguide and give instructions as mentioned in the guide. For this reason an education-protocol is available including a checklist for education and coordination with other health care workers. A videotape will be given to the patient in which exercises and directions are visualised.

The model will be evaluated in an experimental setting with a pre-and posttest and a controlgroup. The preliminary results, the protocols and the self-help-guide will be presented at the symposium.

VI.A.7. The Rheuma School.

M. Brattström, Y. Lindroth. Departments of Rheumatology, Malmö and Lund, Sweden.

A material meant for group education of patients with rheumatoid arthritis (RA).

Treatment of rheumatoid arthritis is concentrated on preventing or limiting physical handicaps and increase the ability of the individual patient to manage daily life. This may be achieved by psychological support, by medical and surgical treatment, by physiotherapy, by principals of joint protection, technical aids etc. Sufficient knowledge within these areas will help the patient to cope with the disease in a more adequate way, perhaps also to change her way of life and behaviour.

The material is meant to be used in group education of RA patients. It consists of -guidelines for tutors -overhead pictures to lecture 1-7 -list of relevant literature -evaluation formulas for each lecture -patient handbooks lecture 1-7 covering: 1) Analysis of relevant problems for the RA patient, how to listen and to communicate ; 2) Medical and surgical treatment ; 3) The nutrition ; 4) How to handle pain and stress ; 5) Principals of joint protection in daily life. Aids and appliances; 6) Crisis and acceptance. Social rights; 7) Practical kitchen training. An extra evening session with relatives and friends is arranged.

The tutor for each part should be the most competent member of the therapeutic team : Doctor, nurse, physiotherapist, occupational therapist, psychologist. All depending on local resources. This education material was used in a clinical trial with 100 Swedish patients treated in Malmö.

VI.A.8. Arthritis education programme development and evaluation.

W. Hill. Physiotherapy Department, St Michael's Hospital, Aylsham, Norwich, Norfolk, NR11 6NA, England. 